\title{
Liking for and consumption of vegetables in European adolescents: Healthy eating, liking, food neophobia and food choice motives
}

\author{
KM Appleton ${ }^{1}$, C Dinnella², S Spinelli², D Morizet ${ }^{3}$, L Saulais ${ }^{4}$, A Hemingway ${ }^{1}$, \\ E Monteleone ${ }^{2}$, L Depezay ${ }^{3}$, FJA Perez-Cueto ${ }^{5}$, and $\mathrm{H}_{\text {Hartwell }}{ }^{1}$ \\ ${ }^{1}$ Bournemouth University, Poole, UK; ${ }^{2}$ University of Firenze, Florence, Italy; \\ ${ }^{3}$ Bonduelle, Villeneuve D'Ascq, France; ${ }^{4}$ Institut Paul Bocuse, Ecully, France; \\ ${ }^{5}$ University of Copenhagen, Copenhagen, Denmark.
}

\begin{abstract}
INTRODUCTION
A high vegetable consumption is associated with many health benefits, yet vegetable consumption across Europe is lower than recommended for health reasons, particularly in adolescents $^{(1)}$

Low vegetable consumption is often attributed to the bitter taste of many vegetables ${ }^{(2,3)}$, yet not all vegetables are bitter-tasting and not all bitter tastes are disliked.

This study investigated the individual characteristics and attitudes associated with the liking for and regular consumption of four bitter vegetables and four sweet vegetables in European adolescents.
\end{abstract}

\section{RESULTS}

Regular consumption of both bitter and sweet vegetables was associated with higher healthy eating habits (smallest $\beta=0.10, p=0.01$ ) and a higher liking for bitter/sweet vegetables respectively (smallest $\beta=0.50, p<0.01$ ) (Table 1 ). Higher liking for bitter vegetables was associated with a lower food neophobia and higher motivations to consume natural foods (smallest $\beta=0.09, p<0.01$ ) (Table 2).

Higher liking for sweet vegetables was associated also with a lower food neophobia, and with higher healthy eating habits and higher motivations to consume foods for sensory reasons (smallest $\beta=0.10, p<0.01$ ) (Table 2).

Some effects of gender and country were also found (Table 3).

Table 3: Consumption (portions) and liking (9-point) per country

\begin{tabular}{|l|l|l|l|l|} 
& $\begin{array}{l}\text { Denmark } \\
(\mathrm{N}=178)\end{array}$ & $\begin{array}{l}\text { UK } \\
(\mathrm{N}=155)\end{array}$ & $\begin{array}{l}\text { France } \\
(\mathrm{N}=206)\end{array}$ & $\begin{array}{l}\text { Italy } \\
(\mathrm{N}=197)\end{array}$ \\
\hline $\begin{array}{l}\text { Consump- } \\
\text { tion bitter }\end{array}$ & $1.2(1.0)$ & $0.9(0.9)$ & $1.2(0.9)$ & $1.1(1.0)$ \\
\hline $\begin{array}{l}\text { Consump- } \\
\text { tion sweet }\end{array}$ & $1.9(1.2)$ & $1.9(1.1)$ & $2.4(1.2)$ & $1.6(1.2)$ \\
\hline Liking bitter & $6.2(1.6)$ & $4.9(1.6)$ & $5.3(1.9)$ & $4.6(1.8)$ \\
\hline Liking sweet & $6.9(1.5)$ & $6.2(1.4)$ & $7.2(1.4)$ & $5.8(1.8)$ \\
\hline
\end{tabular}

\section{DISCUSSION}

These findings suggest that strategies to increase vegetable consumption in adolescents should focus on increasing healthy eating, and increasing vegetable liking. Various techniques can be used to change taste preferences ${ }^{(5)}$.

From our data, increasing liking for both bitter and sweet vegetables may also benefit from strategies to reduce neophobia. Liking for bitter vegetables may benefit also from increasing motivations to consume natural foods, while liking for sweet vegetables may benefit also from increasing healthy eating and from increasing motivations to consume foods for sensory reasons.

\section{METHODS}

Questionnaire data were collected from 736 adolescents (aged 12-19 years) from Denmark $(\mathrm{N}=178)$, France $(\mathrm{N}=206)$, Italy $(\mathrm{N}=197)$ and the UK $(\mathrm{N}=155)$. consumed vegetables - 4 bitter vegetables: broccoli, cauliflower, green salad and spinach $^{(4)}$; 4 sweet vegetables: carrot, peas, sweetcorn and tomato ${ }^{(4)}$; and 3 neither bitter nor sweet vegetables: courgette, green beans, other beans ${ }^{(4)}$. social affluence; and individual healthy eating habits (Adolescent Food Habits Checklist), food neophobia (fear of novel foods) (Food Neophobia Scale), dietary Choice Questionnaire).

Data were analysed using regression -4 models investigated liking and consumption for bitter and sweet vegetables, respectively.
Table 2: Outcomes of the regression analyses for liking

\begin{tabular}{|c|c|c|c|c|}
\hline & Bitter & & Sweet & \\
\hline & Beta & $\mathrm{p}$ & Beta & $p$ \\
\hline Gender & -.09 & $<.01$ & .07 & .03 \\
\hline Age & .06 & .15 & .04 & .40 \\
\hline Denmark & .15 & $<.01$ & .09 & .02 \\
\hline UK & .07 & .08 & .08 & .07 \\
\hline Italy & -.02 & .67 & .31 & $<.01$ \\
\hline FAS Index & .03 & .42 & -.03 & .45 \\
\hline Neophobia & -.25 & $<.01$ & -.11 & $<.01$ \\
\hline AFHC Index & .07 & .06 & .10 & $<.01$ \\
\hline DEBQ & -.03 & .36 & .02 & .50 \\
\hline FCQ - Mood & .07 & .06 & -.01 & .86 \\
\hline FCQ - Sensory & .02 & .62 & .12 & $<.01$ \\
\hline FCQ - Natural & .09 & $<.01$ & .02 & .61 \\
\hline Liking sweet / bitter & .33 & $<.01$ & .35 & $<.01$ \\
\hline
\end{tabular}

\section{REFERENCES}

1. EFSA. Concise Database summary statistics Total population. Website, 2nd March, 2018

2. Brug J, et al. Br J Nutr 2008;99suppl1:S7-S14.

3. Larson NI, et al. Am J Prev Med 2008;35:33-37.

4. Martin C, et al. Food Qual Pref 2014;36:70-80.

5. Wadhera D, et al. Appetite 2015;93:75-84.

Email:k.appleton@bournemouth.ac.uk 